\title{
EDITORIAL
}

\section{Laser surgery for eyelid margin tumors}

\section{Adam Kemeny-Beke}

Department of Ophthalmology, Faculty of Medicine, University of Debrecen, Debrecen, Hungary

Eyelid margin is one of the most challenging anatomic regions in the human body, and eyelid margin tumors raise special difficulties in the field of periocular surgery based on anatomical, histological and aesthetic points of view. Although manually performed surgical excision is the first line choice to remove eyelid margin tumors, previously, of late, lasers have gained popularity with regards to eyelid tumors and especially eyelid margin tumor extraction. There is scarce data with respect to this topic, and most of them are case reports.

Different types of laser apparatus emitting different light beams are available for periorbital laser surgery, i.e. argon laser (Ar, 488-514 nm), erbium:yttrium-aluminiumgarnet laser (Er:YAG, 2790-2940 nm), erbium:yttriumscandium-gallium-garnet laser (Er:YSGG, $2790 \mathrm{~nm}$ ), and carbon dioxide laser with an infrared source $\left(\mathrm{CO}_{2}, 10,600\right.$ $\mathrm{nm})$. Due to the introduction of new-generation technical innovations such as pencil-shaped hand pieces or new working modes, nowadays laser therapy is one of the most widespread forms of periocular surgery.

There are three basic types of interaction between laser beam and tissue: photocoagulation, photodisruption, and photoablation ${ }^{[1]}$; the clinically most utilized of these is the last one. In the course of laser surgery, a zone of coagulative necrosis called lateral thermal damage (LTD) abuts the treated area. Given the fact that the extension of LTD plays an outstanding role in the aesthetic result, the presence of possible side effects should diminish. In order to achieve a more precise ablation zone, the laser pulse duration should be decreased to a period shorter than the thermal relaxation time of soft tissues.

Although newly improved laser sets have several advantages over classic approaches, periocular laser surgery, especially lid margin laser surgery, is not free of complications. Some special features should be taken into consideration, predominantly anatomical and histological issues, tumor localization, and the proximity of the nasolacrimal drainage system.

The main issue concerning every type of surgery is the fact that preoperative diagnosis is the key point, and in cases where this is incorrect, additional treatment will be necessary.

Periorbital laser surgery is high-ranked in ophthalmology as it combines the advantages of laser surgery achieved in dermatology and aesthetic surgery. ${ }^{[2-4]}$ There are several benefits of laser $v s$. traditional excision including less bleeding, tissue destruction, minimal occurrence of dyschromia, etc. Furthermore, there is no postoperative edema and/or ecchymosis, and there are smaller amounts of granulation tissue and, less frequently, fewer unaesthetic scar formations. The laser sharpens excision, evaporation, and fulguration of tissues, all while maintaining hemostasis. One of the best advantages of laser surgery for eyelid margin tumors is its anatomical aspect; since the laser beam keeps the margins in their anatomical position, entropion or ectropion are not to be reckoned with.

Although a more extensive collagen shrinkage can occur due to intense heat compared to manually performed surgical excision, it can be avoided by using appropriate energy fluence ${ }^{[5]}$. Treatment should be started with low energy and then increased, in order to avoid thermal damage.

Several factors may have an impact on the result of eyelid margin laser surgery and some of them may result in side effects

Among the most severe adverse events referred to in the literature concerning periocular laser surgery are severe burns, corneal injuries, or even ocular perforation. A $0.3 \%$ rate of corneal trauma after lower eyelid laser blepharoplasty was reported by the ASAPS/ASPRS Laser Task Force Survey Summary ${ }^{[6]}$. To avoid any corneal damage, stainless steel, or metallic non-refl ctive internal ocular shields are highly recommended in the periorbital areas; by using these materials, no detectable alterations in slit-lamp, pupillary, or funduscopic evaluations after periorbital laser irradiation can be identified. The question of infection could be raised during periocular surgery as, often, an uncovered surface is created during these procedures. Different types of potential infections, i.e., bacterial, viral or fungal can occur in the postoperative period after periocular or eyelid laser surgery. Besides Er:YAG lasers, $\mathrm{CO}_{2}$ lasers have become the current tool for resurfacing and rhytidectomy. Since the aforementioned procedures have been related to increased recovery time mainly in patients with darker skin types, to minimize these imperfections of ablative laser, an ablative fractional photothermolysis laser has been designed, which is able to maintain the favorable clinical efficac of the non-ablative $\mathrm{CO}_{2}$ laser.

Hypertrophic or keloid scar formation, erythema, and

Copyright (C) 2021 Kemeny-Beke A. This is an Open Access article distributed under the terms of the Creative Commons Attribution-NonCommercial 4.0 International License (http://creativecommons.org/licenses/by-nc/4.0/), permitting all non-commercial use, distribution, and reproduction in any medium, provided the original work is properly cited. 
clinical manifestation of vasodilation have also been reported in the literature as some of the most frequent side effects of periocular laser surgery. Pigmentation disorders can also occur following eyelid laser surgery. Hypopigmentation is more frequent than post-inflammatory hyperpigmentation (PIH) after laser treatment, as deep thermal damage eradicates melanocyte population and may unmask a pre-existing vitiligo. This symptom is called the Koebner phenomenon. Some laser surgeons recommend the use of 5-fluorouracil for advancing wound recovery, but this treatment may increase the risk of post-laser hypopigmentation $^{[7]}$. To reduce hyper- or hypopigmentation eff cts, the surgeon has to minimize the laser energy output.

There is a controversy about wearing occlusive dressing in the postoperative period; some authors recommend its application $^{[2]}$, however, others note that this is a risk factor for post-laser surgery's bacterial and fungal infection ${ }^{[8]}$.

To summarize the cognition about eyelid laser surgery, it has to be emphasized that many patients, who are afraid of traditional surgical interventions, are less scared of laser surgery, which is safe, simple, fast, and efficient. Laser surgery is a very useful skill not only in periocular surgery but also in eyelid margin surgery. In order to minimize their functional and aesthetic complications, thorough patient selection, careful and meticulous checkup, and adequate surgeon training are absolutely indispensable.

\section{References}

1. Thall EH. Principles of lasers. In: Yanoff, Duker JS (editors). Ophthalmology. London: Mosby; 1999. p. 251-256.

2. Yates B, Que SK, D'Souza L, Suchecki J, Finch JJ. Laser treatment of periocular skin conditions. Clin Dermatol. 2015; 33(2): 197-206.

3. Trelles MA, Brychta P, Stanek J, Allones I, Alvarez J, et al. Laser techniques associated with facial aesthetic and reparative surgery. Facial Plast Surg. 2005; 21(2): 83-98.

4. Blanco G, Clavero A, Soparkar CN, Patrinely JR. Periocular laser complications. Semin Plast Surg. 2007; 21(1): 074079. doi: $10.1055 / \mathrm{s}-2007-967752$.

5. Fulton JE Jr. Complications of laser resurfacing: Methods of prevention and management. Dermatol Surg 1998; 24(1): 9199. doi: 10.1111/j.1524-4725.1998.tb04059.x.

6. Apfelberg DB. Summary of the 1997 ASAPS/ASPRS Laser Task Force Survey on laser resurfacing and laser blepharoplasty. Plast Reconstr Surg 1998; 101(2): 511-518. doi.org/10.1097/00006534-199802000-00043.

7. Park KY, Oh IY, Moon NJ, Seo SJ. Treatment of infraorbital dark circles in atopic dermatitis with a 2790-nm erbium: yttrium scandium gallium garnet laser: A pilot study. J Cosmet Laser Ther 2013; 15(2): 102-106. doi.org/10.3109/14764172. 2012.759236.

8. Nanni CA, Alster TS. Complications of carbon dioxide laser resurfacing. An evaluation of 500 patients. Dermatol Surg 1998; 24(3): 315-320. doi: 10.1111/j.1524-4725.1998. tb04161.x.

Keywords: periocular laser surgery; eyelid, margin; complication

Citation: Kemeny-Beke A. Laser surgery for eyelid margin tumors. J Surg Dermatol 2021; 6(2): 143. http://dx.doi.org/jsd. v6.i2.143.

Received: $10^{\text {th }}$ June 2021; Published Online: $18^{\text {th }}$ June 2021

*Correspondence to: Adam Kemeny-Beke, Department of Ophthalmology, Faculty of Medicine, University of Debrecen, Nagyerdei krt. 98, H-4032, Debrecen, Hungary; kemenyba@med.unideb.hu 